\section{Macroscopic hematuria: A rare etiology in western countries}

\author{
Bahjat Barakat, ${ }^{1}$ Benedetta Fabbrizio, ${ }^{2}$ \\ Raffaele Pezzilli ${ }^{3}$ \\ 'Emergency Department, Sant'Orsola- \\ Malpighi Hospital, University of Bologna; \\ 2Department of Organ Failure and \\ Transplantation, Sant'Orsola-Malpighi \\ Hospital, University of Bologna; \\ ${ }^{3}$ Department of Digestive system, \\ Sant'Orsola-Malpighi Hospital, University \\ of Bologna, Italy
}

\begin{abstract}
Although schistosomiasis is one of the most prevalent parasitic diseases worldwide, the infection frequently being found in migrants and travelers, its recognition in Italy may be delayed as patients may either present symptoms or be asymptomatic, especially with regard to localization in the bladder, in a similar way to other infectious diseases. We report a case of urinary schistosomiasis in a young African male with persistent hematuria which did not respond to antibiotic treatment administered on suspicion of a urinary bacterial infection. The present case indicates that urinary schistosomiasis should be ruled out, especially in those patients presenting symptoms and coming from areas known to be endemic for helminthiasis. Finally, bladder polyps must be ruled out in cases of migrants with unexplained urinary inflammation associated either with or without hematuria.
\end{abstract}

\section{Introduction}

Although schistosomiasis is one of the most prevalent parasitic diseases worldwide, the infection frequently being found in travelers and migrants, its recognition may be delayed as patients may either be asymptomatic ${ }^{1}$ or present symptoms, especially with regard to localization in the bladder, in a similar way to other infectious diseases. ${ }^{1}$ The European Network for Tropical Medicine and Travel Health carried out a sentinel surveillance study on imported schistosomiasis between 1997 and 2010; in summary: of the 1,465 cases of imported schistosomiasis, direct pathogen detection and serology were the main diagnostic tools applied: one-third of the cases were identified among European travelers, and one half among non-European travelers. Almost all the infections were acquired in Africa and Schistosoma mansoni was identified in $39 \%$ of the cases, whereas Schistosoma haematobium was found in $22 \%$ and about $60 \%$ of the patients presented symptoms. Acute symptoms were reported in $27 \%$ of patients leading to earlier presentation within 3 months. ${ }^{1}$ Clinical presentation is sometimes challenging, especially in its early phases when clinical signs and symptoms are poor and overlap with other diseases. For this reason we believe that the case we present is worth reporting.

\section{Case Report}

A 28 year-old male was admitted to our Emergency Room for a two-month duration hematuria which was occasionally associated with abdominal pain localized in the lower abdomen; he presented no fever. The patient was born and lived in Mali and his past medical history was negative. Due to the presence of hematuria, he was treated with antibiotics on suspicion of a urinary tract infection, but with no benefit. On admission to the Emergency Room, a physical examination was unremarkable: there were no alterations either to the cardiopulmonary system or to the abdomen. He was afebrile, his arterial blood pressure was $110 / 70 \mathrm{mmHg}$ and he had normal oxygen saturation. Blood tests showed an increase in white blood cells $(12.140 \mathrm{mmc})$, an increase in eosinophils $(1120 / \mathrm{mmc})$ and serum concentrations of total $\operatorname{IgE}(4115 \mathrm{IU} / \mathrm{mL}$, upper reference value 180). C-reactive protein was normal as were renal and liver functions. In the urine sediment there were found relatively large ova, measuring from $110 \mu \mathrm{m}$ to $170 \mu \mathrm{m}$ in length and from $40 \mu \mathrm{m}$ to $70 \mu \mathrm{m}$ in width with an elongated ellipsoid shape and a prominent terminal spine. Transabdominal ultrasonography showed no alterations to the liver, spleen, pancreas or kidneys; the bladder showed irregularly thickened walls and two hyperecogenic lesions projecting into the lumen, the first of $1.4 \mathrm{~cm}$ in size localized in the left bladder wall and the second in the posterior bladder wall (Figures 1 and 2); these lesions were avascular to the colorDoppler study. The patient underwent cystoscopy which showed the presence of multiple red and yellow colored solid tumors protruding into the lumen of the bladder (Figure 3) and a stenosis of the left ureter which was treated endoscopically with mechanical dilation by using balloon dilation. Multiple resections of the bladder wall were carried out during the examination and biopsies were also performed; the histology was compatible with Schistosoma Haematobium (Figure 4). In fact, Schistosoma Haematobium has terminal spines whereas Schistosoma Mansoni has
Correspondence: Raffaele Pezzilli, Department of Digestive System, Sant'OrsolaMalpighi Hospital, Via Massarenti 940138 Bologna, Italy.

Tel.: +39.0516364148 - Fax: +39.0516364148. E-mail: raffaele.pezzilli@aosp.bo.it

Key words: Parasitic diseases; schistosoma haematobium; hematuria

Conflict of interest: the authors declare no potential conflict of interest.

Received for publication: 8 September 2017. Revision received: 16 December 2017.

Accepted for publication: 11 January 2018.

This work is licensed under a Creative Commons Attribution 4.0 License (by-nc 4.0).

CCopyright B. Barakat et al., 2017

Licensee PAGEPress, Italy

Emergency Care Journal 2017; 13:7055

doi:10.4081/ecj.2017.7055

lateral spines and Schistosoma Japonicum has no spines at all or small inconspicuous subterminal spines. The search for other parasites in the patient's feces was carried out and was negative. The patient was treated with praziquantel at a dosage of $2400 \mathrm{mg}$ per day for three consecutive days. After one week he was discharged from our hospital in good health with normal urine.

\section{Discussion}

Schistosomiasis is a disease caused by blood trematodes. It has been estimated that 200-300 million people in more than 70 countries are affected by this disease and a further 500-600 million are exposed to the risk of infection: more than 66.5 million people were reported to have been treated for schistosomiasis in $2015 .^{2}$ It is primarily a rural disease affecting agricultural communities and fishermen. There are three important species which affect man: Schistosoma mansoni causes intestinal schistosomiasis and occurs in Africa, Brazil, Venezuela, Madagascar, the Arabian peninsula, the West Indies and Surinam; Schistosoma haematobium causes urinary schistosomiasis and occurs in Africa and the Middle East; Schistosoma japonicum causes intestinal schistosomiasis and occurs in China, Indonesia and the Philippines. The remaining two species infecting humans are Schistosoma intercalatum found in West and Central Africa and Schistosoma mekongi found in the Mekong River Basin. ${ }^{2}$

Cercarial dermatitis (Swimmer's Itch) 


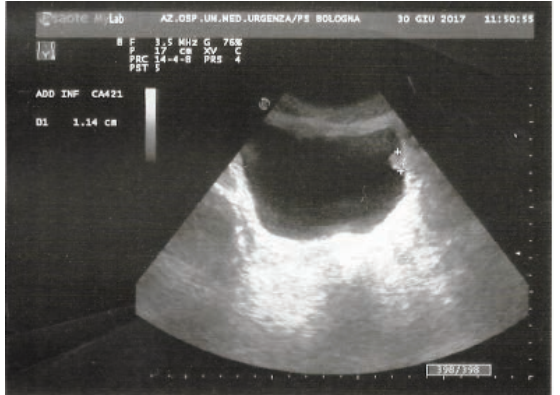

Figure 1. Transabdominal ultrasonography of the bladder showing solid lesion protruding into the lumen indicated by ++ .

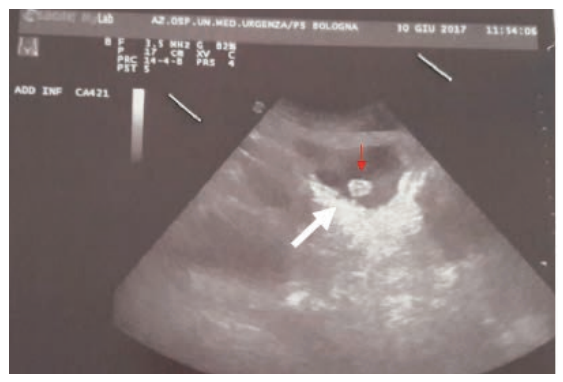

Figure 2. Transabdominal ultrasonography of the bladder showing hyperechoic solid lesion protruding into the lumen (red arrow) and irregularly thickened bladder wall (white arrow).

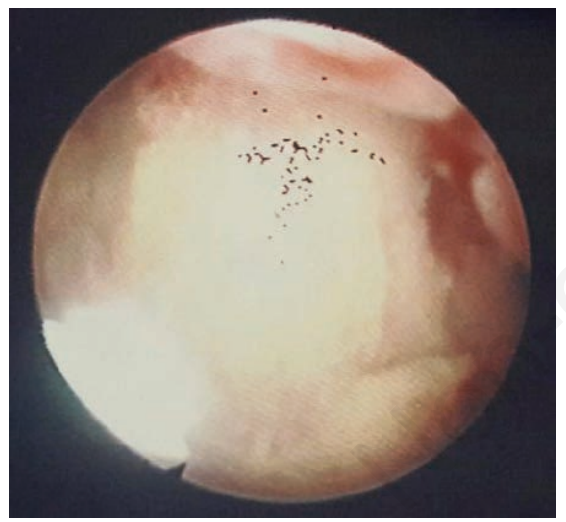

Figure 3. Cystoscopy showing the presence of multiple red and yellow colored solid tumors protruding into the lumen of the bladder.

following skin penetration, results in a maculo-papular rash which can last 36 hours or more. The mature flukes of S. haematobium migrate to the veins surrounding the bladder. After mating, the eggs are laid in the venules of the bladder and many penetrate through the mucosa, enter the lumen of the bladder and are excreted in the urine accompanied by blood. Thus haematuria, as in the case we have reported, and protein-


Figure 4. A) This low-power magnification photo shows a fibrotic urinary bladder wall, with marked vascular congestion and extensive inflammatory infiltrate. Numerous diffuse oval calcification and oval structures are present. B) The oval structures are noncalcified Schistosoma eggs and are associated with a marked acute and chronic inflammatory infiltrate, with numerous eosinophils. No granulomas are evident. C) On high-power examination, the terminal spines of non calcified Schistosoma haematobium eggs are evident. D) In this section, numerous calcified Schistosoma haematobium eggs are present. All figures are $H \& E$-stained slides and magnifications are variable $(2 x, 10 x, 20 x, 40 x)$.

uria are characteristic, though not invariable features of urinary schistosomiasis.

In the chronic infection disease, eggs become trapped in the bladder wall resulting in the formation of granulomata and these alterations can be assessed by ultrasonography. ${ }^{3}$ Following prolonged infection, the ureters may become obstructed in which case the bladder becomes thickened resulting in abnormal bladder function, urinary infection and kidney damage. ${ }^{4}$ Chronic urinary schistosomiasis is sometimes associated with squamous cell bladder cancer and this possibility should be taken into consideration especially when symptoms are of long lasting duration even if the importance of various mechanisms responsible for this association remain unclear. ${ }^{5}$

\section{Conclusions}

In conclusion, our case indicates that bladder polyps due to urinary schistosomiasis should be ruled out, especially in those patients who are symptomatic and come from areas known to be endemic for helminthiasis and there is the need for careful investigations in patients coming from countries in which the infection is endemic.

\section{References}

1. Lingscheid T, Kurth F, Clerinx J, et al. Schistosomiasis in european travelers and migrants: analysis of 14 years TropNet surveillance data. Am J Trop Med Hyg 2017;97:567-74.

2. WHO Fact sheet Schistosomiasis. Available from: http:/www.who.int/ mediacentre/factsheets/fs 115/en/

3. Barda B, Coulibaly JT, Hatz C, Keiser J. Ultrasonographic evaluation of urinary tract morbidity in school-aged and preschool-aged children infected with Schistosoma haematobium and its evolution after praziquantel treatment: a randomized controlled trial. PLoS Negl Trop Dis 2017;11:e005400.

4. Khalaf I, Shokeir A, Shalaby M. Urologic complications of genitourinary schistosomiasis. World J Urol 2012;30:31-8.

5. Honeycutt J, Hammam O, Fu CL, Hsieh $\mathrm{MH}$. Controversies and challenges in research on urogenital schistosomiasisassociated bladder cancer. Trends Parasitol 2014;30:324-32. 